\title{
Open
}

\section{Consanguinity and rare mutations outside of MCCC genes underlie nonspecific phenotypes of MCCD}

\author{
Peter J. Shepard, PhD1, Bruce A. Barshop, MD, PhD ${ }^{1,2}$, Matthias R. Baumgartner, MD ${ }^{3,4}$, \\ John-Bjarne Hansen, $\mathrm{MD}^{5}$, Kristen Jepsen, $\mathrm{PhD}^{1,2}$, Erin N. Smith, $\mathrm{PhD}^{1,2}$ and Kelly A. Frazer, PhD ${ }^{1,2,6}$
}

Purpose: 3-Methylcrotonyl-CoA carboxylase deficiency (MCCD) is an autosomal recessive disorder of leucine catabolism that has a highly variable clinical phenotype, ranging from acute metabolic acidosis to nonspecific symptoms such as developmental delay, failure to thrive, hemiparesis, muscular hypotonia, and multiple sclerosis. Implementation of newborn screening for MCCD has resulted in broadening the range of phenotypic expression to include asymptomatic adults. The purpose of this study was to identify factors underlying the varying phenotypes of MCCD.

Methods: We performed exome sequencing on DNA from 33 cases and 108 healthy controls. We examined these data for associations between either MCC mutational status, genetic ancestry, or consanguinity and the absence or presence/specificity of clinical symptoms in MCCD cases.
Results: We determined that individuals with nonspecific clinical phenotypes are highly inbred compared with cases that are asymptomatic and healthy controls. For 5 of these 10 individuals, we discovered a homozygous damaging mutation in a disease gene that is likely to underlie their nonspecific clinical phenotypes previously attributed to MCCD.

Conclusion: Our study shows that nonspecific phenotypes attributed to MCCD are associated with consanguinity and are likely not due to mutations in the MCC enzyme but result from rare homozygous mutations in other disease genes.

Genet Med advance online publication 6 November 2014

Key Words: consanguinity; 3-methylcrotonyl-CoA carboxylase deficiency; newborn screening; runs of homozygosity

\section{INTRODUCTION}

3-Methylcrotonyl-CoA carboxylase (MCC) deficiency (MCCD) is a recessive disorder caused by homozygous or compound heterozygous mutations in either MCCC1 or MCCC2. Each encodes a subunit of MCC, a biotin-dependent enzyme in the catabolic pathway of leucine. ${ }^{1}$ Clinical expression of MCCD has highly heterogeneous clinical symptoms; some individuals present with developmental delay, intellectual disability, neonatal seizures, ketoacidosis, cerebral edema, coma, or early death, whereas others are asymptomatic into adulthood..$^{2-5}$ Our previous studies have shown that the causative mutations are located throughout the entire coding and some intronic sequences of MCCC1 and MCCC2, resulting in a spectrum of genetic lesions including nonsynonymous, nonsense, splicing, and frameshift mutations. Approximately half of the individuals with MCCD are homozygous for the causative mutation, whereas the other half has compound heterozygous genotypes. Interestingly, neither the type of genetic lesion nor the status of homozygous or compound heterozygous mutation appears to be associated with severity of clinical symptoms. ${ }^{6}$

Although children with MCCD were previously identified during evaluation of developmental disability, metabolic disturbances, or Reye syndrome, ${ }^{7}$ newborn screening by tandem mass spectrometry (targeting 3-hydroxyisovalerylcarnitine as $\mathrm{C} 5 \mathrm{OH}$ carnitine $)^{8}$ in the United States and a number of other countries around the world has resulted in greatly increased ascertainment of MCCD, with an estimated prevalence between 1:41,700 and $1: 84,700 .{ }^{9,10}$ Before its inclusion in newborn screening programs, there were only $\sim 30$ reported cases of MCCD. ${ }^{11}$ Surprisingly, MCCD was found to be the third most common organic acid disorder detected in the newborn screening program in California ${ }^{10}$; however, results of a comparative analysis of case reports with newborn screening data suggest that less than $10 \%$ of affected individuals ever develop minor symptoms and less than 1 to $2 \%$ have a more severe outcome. ${ }^{11}$ When symptoms in an infant are ascertained through newborn screening, it is currently not possible to estimate clinical risk, and thus he/she is frequently administered lifelong treatment regimens including low-protein dietary modification and carnitine supplements. Because symptomatology is rarer than previously thought, prior to newborn screening programs, it is important to determine why some individuals show variable clinical symptoms and others do not. The risk-benefit balance of newborn screening is complicated because there are potential risks involved with too little intervention (not screening or not treating cases that will become symptomatic) or too much intervention (possibly

${ }^{1}$ Department of Pediatrics, Rady Children's Hospital, University of California, San Diego, La Jolla, California, USA; ${ }^{2}$ Clinical and Translational Research Institute, University of California, San Diego, La Jolla, California, USA; ${ }^{3}$ Division of Metabolic Diseases and Children's Research Center, University Children's Hospital Zurich, Zurich, Switzerland; ${ }^{4}$ Zurich Center for Integrative Human Physiology, University of Zurich, Zurich, Switzerland; ${ }^{5}$ Department of Clinical Medicine, University of Troms $\emptyset$, Troms $\emptyset$, Norway; ${ }^{6}$ Institute for Genomic Medicine, University of California, San Diego, La Jolla, California, USA. Correspondence: Kelly A. Frazer (kafrazer@ucsd.edu), Bruce A. Barshop (bbarshop@ucsd.edu) or Erin N. Smith (ens001@ucsd.edu) 
resulting in unnecessary treatment, insurance implications, stigmatization, or promotion of anxiety in asymptomatic cases).

Recent reports have proposed that the attribution of nonspecific symptoms such as intellectual disability, attention deficit disorders, and fatigue to MCCD is questionable ${ }^{10,11}$ and may be caused by genetic variation outside of $M C C C 1$ and MCCC $2 .{ }^{12}$ A number of MCCD patients have been previously reported to have consanguineous parents, ${ }^{12}$ which is associated with a wide variety of health and physical defects attributable to increased numbers of deleterious recessive alleles in the homozygous state. However, using self-reported family histories there was no significant association between parental consanguinity and the presence or absence of symptoms. ${ }^{12}$ Because of the importance of understanding the genetic components that underlie the diverse clinical manifestations of MCCD, in the present study we have used whole-exome sequencing data to directly investigate the role of genetic ancestry and consanguinity.

\section{Cohort selection}

\section{MATERIALS AND METHODS}

All samples were derived from remainders of specimens sent for clinical testing. Although many infants with MCCD that is ascertained through newborn screening appear to be healthy, it is not clear that they will remain asymptomatic. Accordingly, we analyzed 18 asymptomatic mothers who were discovered to have MCCD only by detection of abnormal $\mathrm{C} 5 \mathrm{OH}$-carnitine in the newborn screening sample from their healthy babies in whom the acylcarnitine elevation resolved. With the exception of subject A16, who had been previously reported, ${ }^{6}$ all other samples from asymptomatic individuals in this study were leftover aliquots of de-identified samples from adult females referred to our laboratory for diagnostic enzyme assay with clinical history as indicated. Lymphocytes derived from these 18 individuals were assayed and determined to have a very profound deficiency of MCC. Samples were also studied from 15 patients who were previously reported to be symptomatic, ${ }^{2-4,6,13-22}$ some of whom exhibited specific symptoms that are direct biochemical correlates of defective leucine catabolism, ${ }^{23}$ whereas others were reported to have a wide range of nonspecific symptoms (as outlined in Supplementary Table S1 online). Specific symptoms included ketoacidosis, hypoglycemia, hyperammonemia, coma, and plasma carnitine depletion with gross elevation of hydroxyisovaleryl-carnitine. Nonspecific symptoms, which are not directly related to leucine metabolism, included developmental delay, intellectual disability, seizures, hemiparesis, muscular hypotonia, and multiple sclerosis (Supplementary Table S1 online). We separated the MCCD cases into three groups, with group 1 containing 18 asymptomatic individuals, group 2 containing 5 individuals manifesting specific symptoms, and group 3 containing 10 individuals manifesting only nonspecific symptoms. One hundred eight healthy individuals of Norwegian ancestry from the Tromsø cohor ${ }^{24}$ were used as controls. The University of California, San Diego, Institutional Review Board (Human Research Protection Program) exempted the project under 45 CFR 46 subpart A $\$ 46.101$ b, category 4.

\section{Whole-exome sequencing}

DNA $(2.5 \mu \mathrm{g})$ was fragmented to $~ 175 \mathrm{bp}$ using sonification (Covaris, Woburn, MA), fragment ends were repaired, and adaptors including sample index barcodes were ligated. The resulting DNA libraries were amplified by six cycles of PCR, 500 ng enriched using SureSelect Human All v4 kit (Agilent, Santa Clara, CA) to capture $51 \mathrm{Mb}$ of target sequence, ${ }^{25}$ and sequenced (paired-end 100-bp reads) using a Illumina HiSeq 2000 (Illumina, San Diego, CA) ( $\sim 100$ million reads per sample; $\sim 30 \times$ coverage). Reads were aligned to the human reference genome (hg19) using BurrowsWheeler Aligner (BWA ${ }^{26}$; read pairs identified as duplicates by Picard MarkDuplicates (http://sourceforge.net/projects/picard/) were removed. Using Genome Analysis Toolkit (GATK), ${ }^{27}$ reads were realigned around insertion/deletion sites and base quality scores were recalibrated. Genotypes were called in all 140 samples simultaneously using GATK and filtered using the variant quality score recalibration protocol.

Exome sequence data are available through $\mathrm{dbGaP}$ accession phs000776. http://www.ncbi.nlm.nih.gov/projects/gap/cgi-bin/ study.cgi?study_id=phs000776.v1.p1.

\section{Identity by descent estimation}

Identity by descent was estimated using PLINK (v1.07) ${ }^{28}$ using autosomal single-nucleotide polymorphisms (SNPs) with genotyping call rates $>0.99$, minor allele frequency $>5 \%$, and a per-sample call rate $>80 \%$. Pruning was performed to retain SNPs in linkage equilibrium at $r^{2}$ value $<0.2$.

\section{Ancestry estimation}

Ancestry was estimated as previously described ${ }^{29}$ using the reference population of 1,445 unrelated participants of the 1000 Genomes Project (1KG) comprising five superpopulation groups (African, Asian, South Asian, European, and American admixed). Genotypes of these individuals on the Illumina Omni 2.5 array (Illumina, San Diego, CA) were downloaded (29 August 2012), and variants were linked to dbSNP 135 identifiers using GATK (there were 41,572 SNPs shared between the Omni 2.5 array and the exome sequencing data set). To identify SNPs informative for ancestry, informativeness $\left(I_{\mathrm{n}}\right)$ was calculated across the five superpopulation groups, and markers were chosen in order of $I_{\mathrm{n}}$ that were in low linkage equilibrium $\left(r^{2}<0.2\right)$ with previously chosen markers within $1 \mathrm{Mb}$. SNPs with positive informativeness $(n=29,973)$ were used to cluster the genotypes of each MCCD case with the $1 \mathrm{KG}$ participants using multidimensional scaling in PLINK v1.07. To identify the most similar superpopulation for each MCCD case, a linear discriminant model was created based on the top 20 multidimensional scaling components using linear discriminant analysis (lda command in MASS package ${ }^{30}$ in R) with the $1 \mathrm{KG}$ individuals as a training set.

\section{Identification and analysis of runs of homozygosity}

We identified long stretches of homozygous DNA (runs of homozygosity (ROHs)) using PLINK v1.07 (--homozyg) with a sliding window of 100 SNP length and a minor allele frequency criterion of 0.05 across MCCD and Tromsø individuals. 
Table 1 Mutation status of MCCC1 and MCCC2 genes and clinical data of 35 MCCD patients

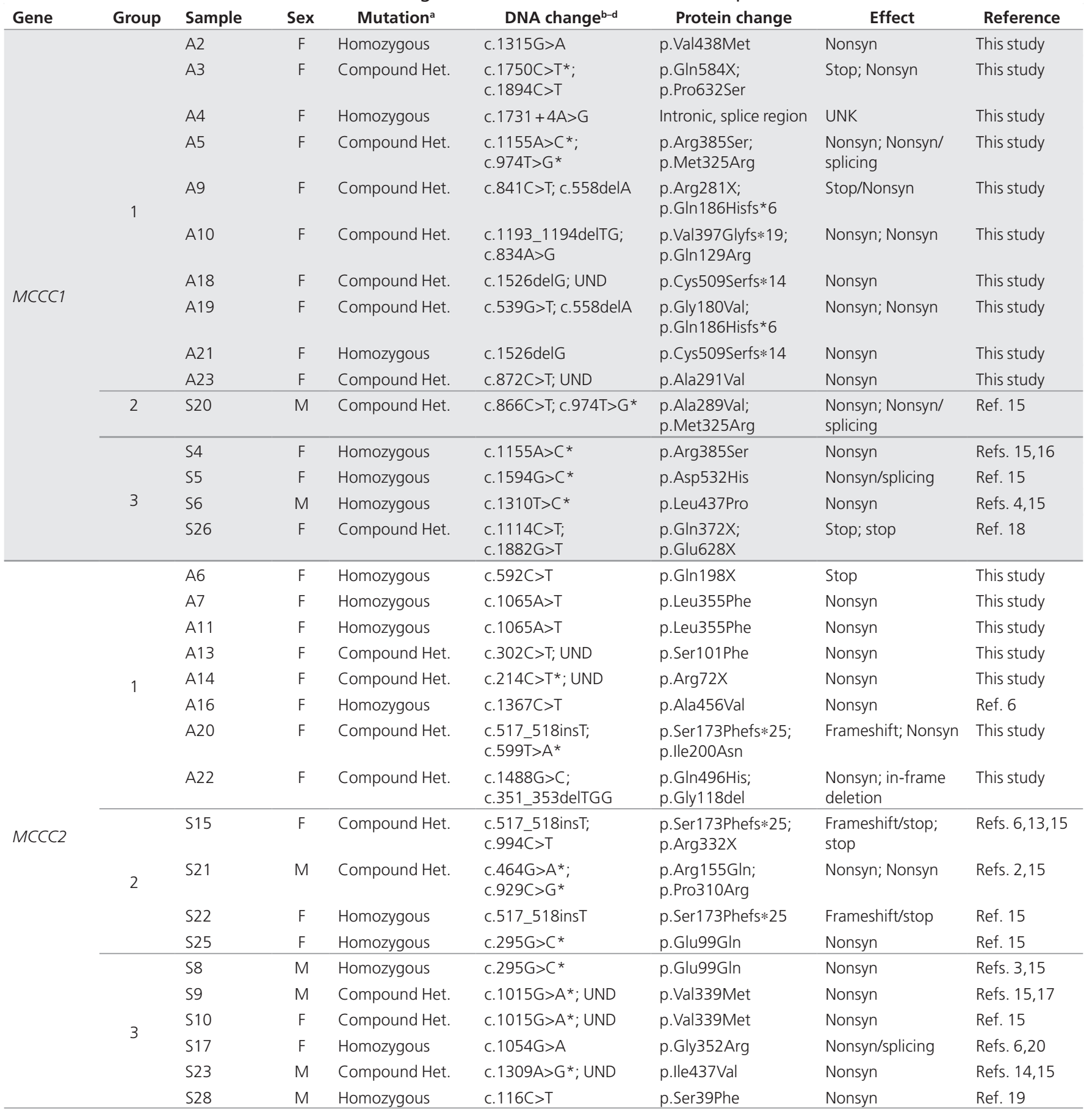

F, female; fs, frameshift; frameshift/stop, frameshift mutation that also results in a downstream stop codon; Het., heterozygous; M, male; MCCD, 3-methylcrotonyl-CoA carboxylase deficiency; Nonsyn, nonsynonymous; nonsyn/splicing, nonsynonymous mutation that also disrupts mRNA splicing; UND, undetermined; UNK, unknown.

aMutation type: homozygous, both alleles are non-hg19 reference genome; compound Het., two heterozygous non-hg 19 reference genome alleles. blf only one allele is found in a compound heterozygote, then the second allele is shown as "UND" in the DNA change column. ${ }^{* *}$ Present in dbSNP at frequency less than 0.0015. ${ }^{\mathrm{V}}$ Variants are reported with reference to transcripts NM_020166.3 for MCCC1 and NM_022132.4 for MCCC2.

The window threshold to call ROHs was $250 \mathrm{~kb}$, and default values for the number of heterozygous SNPs $(=1)$ and number of missing SNPs (=5) allowed in the ROH were used. To ensure that locally low SNP density did not spuriously increase the length of an ROH, PLINK v1.07 default values were used with a minimum SNP density of $50 \mathrm{~kb}$ and a maximum gap between two consecutive SNPs of $1,000 \mathrm{~kb}$. To determine if the numbers of individuals with large ROHs in the three MCCD groups were statistically different, for each individual all regions larger than $1,600 \mathrm{~kb}$ were summed, and a Wilcoxon rank sum test was used 

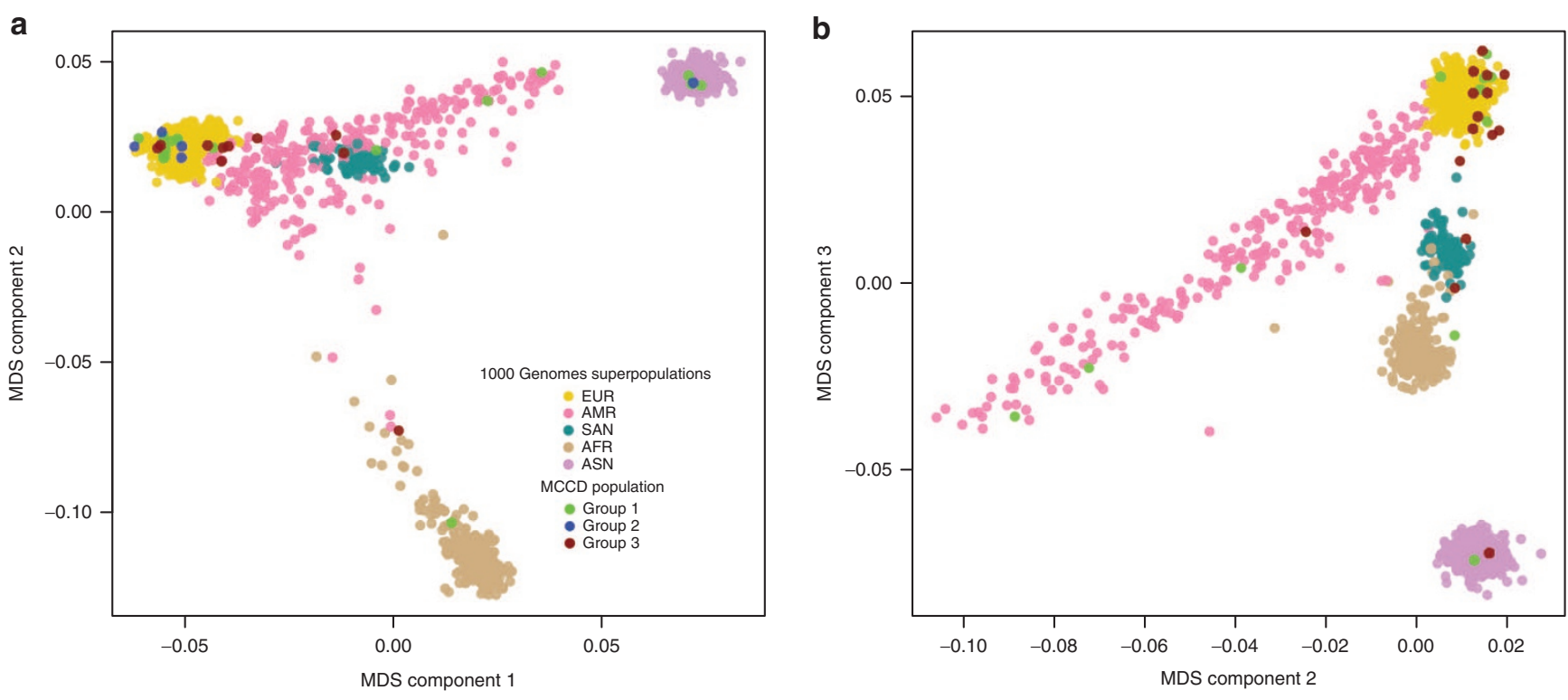

Figure 1 Genetic clustering of participants in the 1000 Genomes Project and MCCD individuals. One thousand four hundred forty-five unrelated individuals from the 1000 Genomes Project and 33 individuals from the MCCD population were clustered on genotype profiles using multidimensional scaling (MDS) and plotted according to their final scores on the (a) first two dimensions and (b) the second and third dimensions. European (EUR), African (AFR), and Asian (ASN) 1000 Genomes Project superpopulation groups are clearly differentiated in the first two dimensions, whereas South Asian (SAN) and American admixed (AMR) groups are overlapping, reflecting their historical European and Asian ancestry. The individuals in the SAN group cluster together. The AMR group is broadly distributed, indicating that some individuals are genetically more similar to the EUR group than others to either the ASN or AFR groups. In the second and third dimensions, SAN and AMR are clearly and distinctly identifiable. MCCD, 3-methylcrotonyl-CoA carboxylase deficiency.

to perform pairwise comparisons between groups 1, 2, and 3 and the Norwegian controls.

\section{Identification and annotation of high-impact variants in OMIM genes}

Variant Call Format (VCF) files were annotated using SnpEff software $^{31}$ and only high-impact (missense, frameshift, or splice site mutations) or moderate-impact (nonsynonomous mutations) variants were kept. Variants were further required to satisfy four criteria: (i) in a homozygous region $\geq 1.6 \mathrm{Mb}$ in length; (ii) in a gene with an OMIM entry (http://omim. org/); (iii) either not present or at a frequency less than $1 \%$ in dbSNP (v137); and (iv) occurring in the exon or splice site of the OMIM gene according to the UCSC Genome Browser mRNA track. For Table 1, gene variants were annotated relative to transcripts NM_020166.3 for MCCC1 and NM_022132.4 for MCCC2 using Variant Effect Predictor (VEP). ${ }^{32}$ Gene variants are reported in accordance with the Human Genome Variation Society (http://www.hgvs.org/).

\section{RESULTS}

Whole-exome sequencing reveals no association between the type of genetic lesion or the status of homozygous/ compound heterozygous mutations and the severity of clinical symptoms

To examine the genetic components underlying the varying clinical phenotypes associated with MCCD, we performed whole-exome sequencing identifying 274,496 and 307,935 variants (single-nucleotide variants and insertion/deletions combined) in the 33 cases and 108 controls, respectively. We first analyzed the mutational spectrum of the MCCC1 and MCCC2 genes in the 33 cases and observed that 15 individuals had mutations in MCCC1 and 18 in MCCC2 (Table 1). Fifteen cases were homozygous (6 for MCCC1 and 9 for MCCC2), and 18 were compound heterozygous ( 9 for MCCC1 and 9 for MCCC2). We observed 35 independent mutations, of which 8 mutations were observed more than once, resulting in the following patient pairs sharing a mutation: A5 and S20, A7 and A11; A9 and A19, A18 and A21; S8 and S25; S9 and S10; S4 and A5; S15, S22, and A20 (Table 1). Of the 35 mutations, 14 are reported in dbSNP (v137) with frequencies $<0.0015$, which is consistent with MCCC1 and MCCC2 null mutations being very rare in the population. For one case (A4) we identified a homozygous mutation $4 \mathrm{bp}$ after an intron/exon boundary in $M C C C 1$, suggesting this mutation may affect splicing. For seven cases we identified only one mutant allele (A13, A14, A18, A23, S9, S10, and S23) and inferred that they were compound heterozygotes because all individuals were shown to have enzyme deficiency, which is not the case when only a single allele is mutated. ${ }^{33}$ Consistent with previous reports, ${ }^{6,12}$ there was no apparent association between the type of genetic lesion or the status of homozygous or compound heterozygous mutations and the absence or presence/severity of clinical symptoms.

MCCD cases that share the same mutation are likely to have inherited the variant from a common ancestor

For the MCCD cases that shared a common mutation, we checked for relatedness to determine if the mutations were 
a

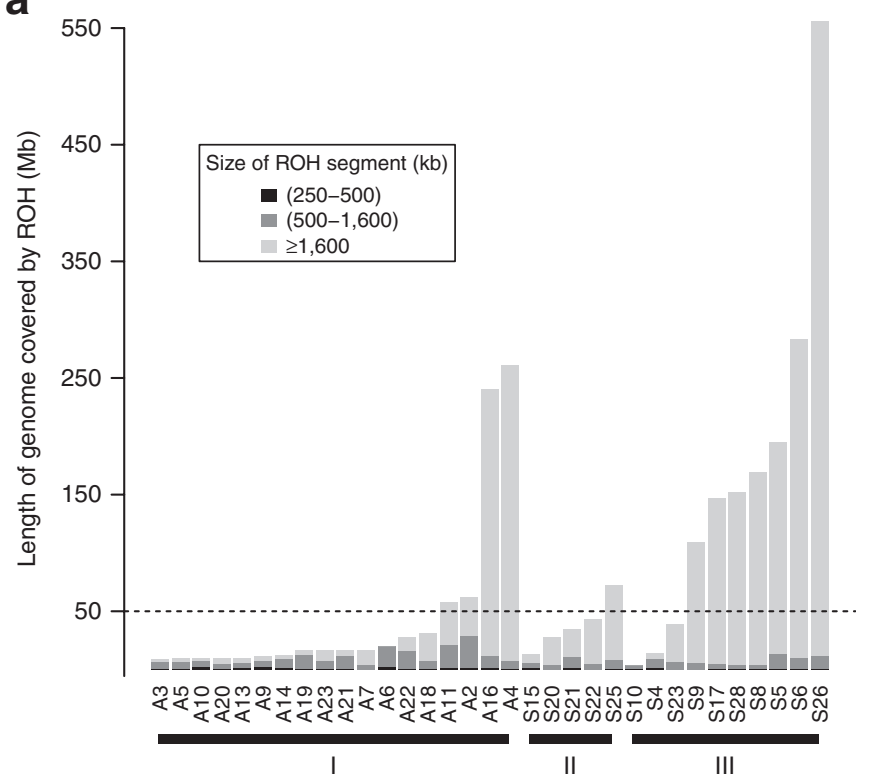

b

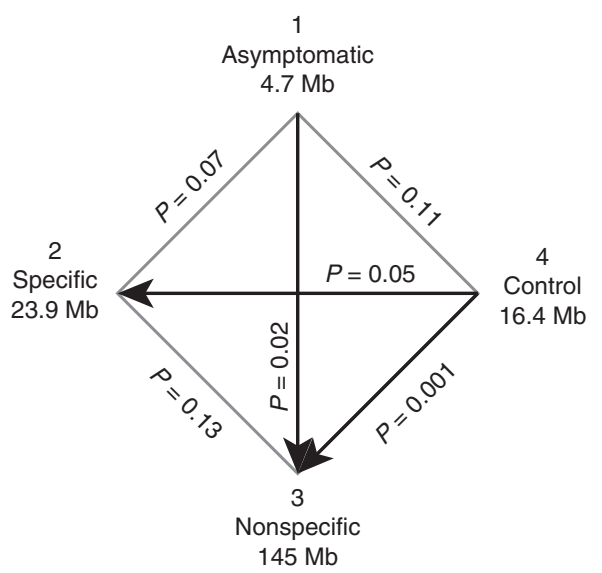

Figure 2 Comparison of amount and type of ROH for MCCD groups 1, 2, and 3. (a) For each individual, the summed totals of ROHs for the three classes (250-500 kb, 500-1,600 kb, >1,600 kb) are shown for MCCD individuals organized by symptom group. The dashed line indicates the expected amount of homozygosity that would result in the union of second cousins. (b) Pairwise $P$ values from the comparison of total ROHs $>1,600 \mathrm{~kb}$ between each MCCD group and Tromsø control group using a Wilcoxon rank sum test. Below each group is the median total length of $\mathrm{ROH}$ segments $>1,600 \mathrm{~kb}$ for the individuals in each group. MCCD, 3-methylcrotonyl-CoA carboxylase deficiency; $\mathrm{ROH}$, run of homozygosity.

inherited from a common ancestor. We estimated the proportion of the genome shared identically by descent using PLINK (PI_HAT value). We did not observe individuals related at the level of second-degree relatives or closer because all pairs of individuals were reported as having PI_HAT $<0.25$. Half of the pairs of individuals who shared a mutation had cryptic relatedness: A18 and A21 (MCCC1 c.1526delC mutation) had a PI_HAT $=0.13$, indicating third-degree relatives; S4 and A5 (MCCC1 c.1155A>C mutation) had a PI_HAT of 0.09, indicating fourth-degree relatives; A5 and S20 (MCCC1 c.974T>G mutation) have a PI_HAT of 0.09 , indicating fourth-degree relatives; A9 and A19 (MCCC1 c.558delA mutation) had a PI_HAT of 0.07 , indicating fourth-degree relatives; and S22, S15, and A20 (MCCC2 c.517_518insT mutation) had pairwise PI_HAT of 0.09 (Supplementary Table S2 online). For the three pairs of MCCD cases that shared an MCCC2 mutation but did not have cryptic relatedness, we examined the genotypes of 36 SNPs (minor allele frequency $>5 \%$ ) in the $265-\mathrm{kb}$ interval encompassing the MCCC2 gene for identity by state (Supplementary Table S3 online). If the mutation had been inherited from the same distant relative, one would expect them to share not only the variant but also the surrounding chromosomal region. Cases S25 and S8 (MCCC2 c.295G>C mutation) had identical genotypes at all 36 SNPs, indicating identity by descent. Cases A7 and A11 (MCCC2 c.1065A>T mutation) had identical genotypes at 35 of the $36 \mathrm{SNPs}$, indicating identity by descent. S9 and S10 (both compound heterozygotes for MCCC2 c.1015G $>$ A mutation) had dissimilar genotypes in the proximal part of the interval but had three SNPs (spanning $5.5 \mathrm{~kb}$ ) immediately proximal to the mutation and 21 SNPs (spanning $~ 800$ $\mathrm{Kb})$ distal to the mutation in which one allele was shared in common, suggesting the mutation arose only once in a distant ancestor. Our data demonstrate that MCCD cases that share the same mutation are most likely to have inherited the variant from a common ancestor.

\section{Genetic ancestry does not seem to be an important determinant of the diverse clinical manifestations}

To determine whether ethnicity plays a role in the manifestation of different clinical phenotypes, we estimated the genetic ancestry of each MCCD case using the 1KG superpopulations (see Materials and Methods). We visualized ancestry estimation for all cases by plotting the first versus second multidimensional scaling components (Figure 1a) and the second versus third components (Figure 1b). We had self-reported ancestry for 11 individuals and observed high agreement between reported ancestry and clustering with expected $1 \mathrm{KG}$ superpopulations (Supplementary Table S4 online). Additionally, the seven pairs of individuals who inherited the same mutation from a common ancestor had similar estimated genetic ancestry. We observed that there are approximately equal numbers of asymptomatic (group 1) and symptomatic MCCD individuals (groups 2 and 3) assigned to each of the five $1 \mathrm{KG}$ superpopulations. Thus, genetic ancestry does not seem to be an important determinant of the diverse clinical manifestations observed among individuals with MCCD.

\section{Association of consanguinity with MCCD clinical phenotypes}

To examine whether consanguinity is associated with MCCD clinical phenotypes, for each case we assessed the amount and characterized the length of autosomal DNA segments that were homozygous (runs of homozygosity, ROHs). ROHs are common in human genomes and represent segments in which identical haplotypes are inherited from each parent. Pemberton et al. ${ }^{34}$ recently classified ROHs by length: short ROHs, which reflect 
Table 2 Mutations underlying the nonspecific clinical phenotypes of MCCD cases

\begin{tabular}{|c|c|c|c|c|c|c|c|c|}
\hline Sample & Chromosome & Position & dbSNP ID & $\begin{array}{c}\text { Global } \\
\text { minor allele } \\
\text { frequency }^{\mathrm{a}}\end{array}$ & $\begin{array}{l}\text { Reference } \\
\text { allele }\end{array}$ & $\begin{array}{c}\text { Alternate } \\
\text { allele }\end{array}$ & Effect & $\begin{array}{c}\text { Gene } \\
\text { symbol }\end{array}$ \\
\hline S9 & chr11 & 68703767 & - & 0 & $C$ & CA & Frameshift & IGHMBP2 \\
\hline S28 & chr 5 & 125880679 & - & 0 & GC & G & Frameshift & $A L D H 7 A 1$ \\
\hline S8 & chr1 & 227171824 & rs144147839 & 0.00022 & A & G & Nonsynonomous & $A D C K 3$ \\
\hline S26 & chr17 & 26727721 & rs5819844 & 0.00138 & $\mathrm{GA}$ & G & Frameshift & $S L C 46 A 1$ \\
\hline
\end{tabular}

MCCD, 3-methylcrotonyl-CoA carboxylase deficiency.

aThe global minor allele frequency is reported from dbSNP v. 137 and is derived from the 1000 Genomes Project phase I data.

Table 3 OMIM entry disease descriptions matched with the symptoms of the corresponding MCCD case

\begin{tabular}{|c|c|c|c|c|}
\hline Sample & Symptoms ${ }^{a}$ & $\begin{array}{l}\text { OMIM } \\
\text { number }\end{array}$ & OMIM title & Matching terms \\
\hline S17 & $\begin{array}{l}\text { Failure to thrive at age } 7 \text { months, frequent watery } \\
\text { diarrhea, fatigue }\end{array}$ & 222470 & $\begin{array}{l}\text { TRICHOHEPATOENTERIC } \\
\text { SYNDROME } 1 ; \text { THES1 }\end{array}$ & $\begin{array}{l}\text { Failure to thrive, frequent watery } \\
\text { diarrhea }\end{array}$ \\
\hline S28 & $\begin{array}{l}\text { Neonatal seizure onset, unresponsive to standard } \\
\text { anticonvulsants, status epilepticus }\end{array}$ & 107323 & $\begin{array}{l}\text { EPILEPSY, PYRIDOXINE- } \\
\text { DEPENDENT }\end{array}$ & $\begin{array}{l}\text { Neonatal seizure onset, } \\
\text { unresponsive to standard } \\
\text { anticonvulsants, status epilepticus }\end{array}$ \\
\hline S8 & $\begin{array}{l}\text { Developmental delay, muscular hypotonia, } \\
\text { progressive seizures, died from circulatory failure } \\
\text { after prolonged epileptic seizures }\end{array}$ & 606980 & $\begin{array}{l}\text { AARF DOMAIN-CONTAINING } \\
\text { KINASE 3; ADCK3 }\end{array}$ & $\begin{array}{l}\text { Developmental delay, progressive } \\
\text { seizures }\end{array}$ \\
\hline S26 & $\begin{array}{l}\text { Psychomotor developmental delay, encephalopathy } \\
\text { at } 5 \text { years of age, hemiparesis at } 13 \text { years of age } \\
\text { consistent with multiple sclerosis course, neutropenia }\end{array}$ & 611672 & $\begin{array}{l}\text { SOLUTE CARRIER FAMILY } \\
46 \text { (FOLATE TRANSPORTER), } \\
\text { MEMBER } 1\end{array}$ & $\begin{array}{l}\text { Delayed motor development, } \\
\text { delay in myelination, hemiplegic, } \\
\text { leukopenia }\end{array}$ \\
\hline
\end{tabular}

MCCD, 3-methylcrotonyl-CoA carboxylase deficiency.

asymptoms as reported by treating physician (references in Table 1).

ancient haplotype blocks; intermediate ROHs, which reflect background relatedness attributable to a population bottleneck; and large ROHs, attributable to recent parental relatedness. For each case, $\mathrm{ROH}$ s were categorized into three size classes: short regions $(250-500 \mathrm{~kb})$, medium regions $(500-1,600 \mathrm{~kb})$, and large regions $>1,600 \mathrm{~kb}$ (Figure 2a). Four (22\%) group 1 cases, one $(20 \%)$ group 2 case, and seven (70\%) group 3 cases have $\mathrm{ROHs}$ at or greater than the level anticipated for individuals who are the product of second cousin unions. We performed all pairwise comparisons among the three MCCD groups to test whether the groups differed by their total length of large ROHs ( $>1,600 \mathrm{~kb}$ ) using a Wilcoxon rank sum test (Figure $2 \mathbf{b}$ ). We show that MCCD group 3 has significantly higher levels of homozygosity than MCCD group $1(P=0.02)$. Although the data suggest that group 3 has higher median levels of homozygosity than group 2 and that group 1 had lower median levels than group 2, the test failed to achieve significance $(P=0.13$ and $P=0.07$, respectively), possibly because of a small sample sizes. Recent studies show that ROHs are more common and longer than expected in unrelated individuals from outbred populations. ${ }^{35,36}$ For this reason we analyzed the 108 controls (Supplementary Figure S1 online), which revealed that only $5(5 \%)$ of these individuals had ROHs at the level consistent with being the product of a second cousin union. Comparisons of each MCCD group with the healthy control group using the Wilcoxon rank sum test demonstrate that group 2 and group 3 MCCD cases had higher median homozygosity than the controls, but only group 3 showed a significant difference $(P=0.001)$. Our results show that group 3 cases are significantly more likely to be inbred compared with cases that are asymptomatic and healthy controls.

\section{Rare mutations outside of MCCC genes underlie nonspecific phenotypes of MCCD}

We hypothesized that group 3 cases harbor homozygous recessive mutations in disease genes other than MCCC1 or MCCC2 that are responsible for their nonspecific clinical phenotypes. We performed a genome-wide search for rare homozygous variants in large $\mathrm{ROHs}$ with predicted high or moderate impact on the function of a gene with an OMIM entry. For two patients, we observed novel mutations in disease genes and a striking number of the patients' clinical symptoms matching the symptoms described in the OMIM entry. In S9, a 1-bp homozygous deletion results in a premature stop codon in immunoglobulin mu binding protein 2 (IGHMBP2) (Table 2). Homozygous mutations in IGHMBP2 are known to cause spinal muscular atrophy type 1, which has clinical features matching those of S9 (Table 3). In S28, we identified a 1-bp homozygous deletion in aldehyde dehydrogenase 7 family, member A1 (ALDH7A1), which may better explain the reported neonatal seizures and 
medically resistant status epilepticus in this patient. For three patients, we identified rare variants (minor allele frequency $<0.0015)$ that result in nonsynonomous substitutions or frameshifts in OMIM disease genes. Although these mutations are in dbSNP, they occur less frequently than the CFTR p.Phe508del mutation (rs121909001; minor allele frequency $\sim 0.015$ ), which is the leading cause of cystic fibrosis. For S17, we observed a homozygous nonsynonomous variant in tetratricopeptide repeat domain 37 (TTC37), which may underlie the reported frequent watery diarrhea, fatigue, and failure to thrive in this individual. In S8, a homozygous nonsynonomous variant in aarF domain containing kinase 3 (ADCK3) may explain the reported progressive seizures and developmental delay. Finally, in S26 a homozygous variant resulting in a frameshift in solute carrier family 46 (folate transporter), member 1 (SLC46A1), may result in the reported dysmyelination and hemiparesis. Thus, for $50 \%$ of the MCCD cases in group 3, we observed a homozygous damaging novel mutation or rare variant in a disease gene that likely explains their clinical symptoms independently of their mutations in $M C C C 1$ or $M C C C 2$.

\section{DISCUSSION}

The recommendation from the American College of Medical Genetics and Genomics to include MCCD in the newborn screening core panel ${ }^{37}$ was based on availability and perceived necessity of treatment and good understanding of the natural history of the disease. Our study suggests that many of the nonspecific symptoms attributed to MCCD may not be caused by mutations in MCC, but rather are caused by mutations in other disease genes. Although having multiple rare recessive mutations is unusual, the probability of having any rare recessive disease is increased by consanguinity. Thus, it is not unusual that patients from consanguineous unions affected with MCCD also appear to be more likely to carry other rare mutations. Our results together with findings of other studies ${ }^{9,33,38}$ suggest that the clinical consequences of MCCD may be less pervasive than previously believed, and when present may be limited to specific effects of defective leucine catabolism. Further studies are needed to assess the value of providing treatment to asymptomatic individuals with MCCD who are identified through newborn screening.

Although our results help to explain the difference between MCCD patients with nonspecific and specific symptoms, they do not address why there are phenotypic differences between asymptomatic MCCD individuals and MCCD patients with specific symptoms. The biochemical context of MCC in leucine catabolism might arguably render its deficiency particularly likely to escape phenotypic expression. Substrate accumulation seems to be well tolerated to very high levels, and excretion of 3-methylcrotonylglycine, 3-hydroxyisovaleric acid, and 3-hydroxyisovalerylcarnitine is efficient (provided that there is no depletion of carnitine). Also, the impact of product limitation may be minimal because the product of MCC, 3-methylglutaconylCoA, can be generated by reversal of 3-methylglutaconyl-CoA hydratase, and the subsequent product, hydroxymethylglutaryl-CoA, may be alternatively formed from acetoacetate derived from fatty acid oxidation. Therefore, to observe a disease phenotype, it may be that mutations other than those in $M C C C 1$ and $M C C C 2$ are needed. Another possibility is that environmental factors (e.g., infection, catabolic stress) trigger specific symptoms in some MCCD patients. Further studies using cases specifically determined not to be inbred might help resolve the difference between asymptomatic MCCD cases and MCCD cases with specific symptoms.

Our study has implications for current undiagnosed disease studies that are attempting to correlate phenotypes to specific genotypes through large-scale sequencing. Because individuals from consanguineous unions carry rarer recessive mutations that could cause genetic diseases, these individuals may be more likely to be recruited to very rare disease studies. For the same reason, they would also be more likely to have phenotypes that could be due to multiple loci. Therefore, care must be taken in extrapolating that a mutation that explains a portion of the clinical phenotype is causal for the entire complex phenotype. Patients with symptoms beyond the usual spectrum of disease for a given disorder should be evaluated for other contributing alleles and putative phenotypic modifiers. Additionally, inbred individuals are often purposely used to identify disease genes due to the ease of mapping autosomal recessive disorders by identifying regions of the genome that are homozygous in all affected individuals. ${ }^{39,40}$ Our results suggest that both genotypephenotype correlative sequencing projects and homozygosity mapping studies should take into account the level of inbreeding in individuals when attributing the effects of a shared mutation on a phenotypically heterogeneous group.

\section{SUPPLEMENTARY MATERIAL}

Supplementary material is linked to the online version of the paper at http://www.nature.com/gim.

\section{ACKNOWLEDGMENTS}

The authors acknowledge Yang Dai for excellent technical assistance and Elizabeth Ferrer-Perez and Céline Bürer (Zurich) for collection and organization of the samples. The authors are grateful to Olivier Harrismendy and Shawn Yost for productive discussions regarding variant calling methods. This study was supported by National Institutes of Health grant 5R03HD69983-2 (to B.A.B.) and a grant from the Swiss National Science Foundation (to M.R.B., SNFS-3200AO-109219/1).

\section{DISCLOSURE}

The authors declare no conflict of interest.

\section{REFERENCES}

1. Gibson KM, Elpeleg O, Morton DH, Wappner R. Disorders of leucine metabolism. In: Blau N, Duran M, Blaskovics M, Gibson KM (eds). Physician's Guide to the Laboratory Diagnosis of Metabolic Diseases. Springer: Berlin, Germany, 2003:165-189.

2. Beemer FA, Bartlett K, Duran M, et al. Isolated biotin-resistant 3-methylcrotonyl-CoA carboxylase deficiency in two sibs. Eur J Pediatr 1982; 138:351-354. 
3. Bannwart C, Wermuth B, Baumgartner R, Suormala T, Weismann UN. Isolated biotin-resistant deficiency of 3-methylcrotonyl-CoA carboxylase presenting as a clinically severe form in a newborn with fatal outcome. $J$ Inherit Metab Dis 1992;15:863-868.

4. de Kremer RD, Latini A, Suormala T, et al. Leukodystrophy and CSF purine abnormalities associated with isolated 3-methylcrotonyl-CoA carboxylase deficiency. Metab Brain Dis 2002;17:13-18.

5. Baykal T, Gokcay GH, Ince Z, et al. Consanguineous 3-methylcrotonyl-CoA carboxylase deficiency: early-onset necrotizing encephalopathy with lethal outcome. J Inherit Metab Dis 2005;28:229-233.

6. Dantas MF, Suormala T, Randolph A, et al. 3-Methylcrotonyl-CoA carboxylase deficiency: mutation analysis in 28 probands, 9 symptomatic and 19 detected by newborn screening. Hum Mutat 2005;26:164.

7. Sweetman L, Williams J. Branched chain organic acidurias. In: Scriver $C R$, Beaudet AL, Sly WS, et al. (eds). The Metabolic and Molecular Bases of Inherited Disease. McGraw-Hill: New York, 2001:2125-2164.

8. Koeberl DD, Millington DS, Smith WE, et al. Evaluation of 3-methylcrotonylCoA carboxylase deficiency detected by tandem mass spectrometry newborn screening. J Inherit Metab Dis 2003;26:25-35.

9. Feuchtbaum L, Carter J, Dowray S, Currier RJ, Lorey F. Birth prevalence of disorders detectable through newborn screening by race/ethnicity. Genet Med 2012;14:937-945.

10. Lam C, Carter JM, Cederbaum SD, et al. Analysis of cases of 3-methylcrotonyl CoA carboxylase deficiency (3-MCCD) in the California newborn screening program reported in the state database. Mol Genet Metab 2013;110:477-483.

11. Stadler SC, Polanetz R, Maier EM, et al. Newborn screening for 3-methylcrotonyl-CoA carboxylase deficiency: population heterogeneity of MCCA and MCCB mutations and impact on risk assessment. Hum Mutat 2006;27:748-759.

12. Grünert SC, Stucki M, Morscher RJ, et al. 3-Methylcrotonyl-CoA carboxylase deficiency: clinical, biochemical, enzymatic and molecular studies in 88 individuals. Orphanet J Rare Dis 2012;7:31

13. Gitzelmann R, Steinmann B, Niederwieser A, Fanconi S, Suormala T, Baumgartner R. Isolated (biotin-resistant) 3-methylcrotonyl-CoA carboxylase deficiency presenting at age 20 months with sopor, hypoglycaemia and ketoacidosis. J Inherit Metab Dis 1987;10:290-292.

14. Mourmans J, Bakkeren J, de Jong J, et al. Isolated (biotin-resistant) 3-methylcrotonyl-CoA carboxylase deficiency: four sibs devoid of pathology. J Inherit Metab Dis 1995;18:643-645.

15. Baumgartner MR, Almashanu S, Suormala T, et al. The molecular basis of human 3-methylcrotonyl-CoA carboxylase deficiency. J Clin Invest 2001;107:495-504.

16. Steen $C$, Baumgartner ER, Duran $M$, et al. Metabolic stroke in isolated 3-methylcrotonyl-CoA carboxylase deficiency. Eur J Pediatr 1999;158:730-733.

17. Wiesmann UN, Suormala T, Pfenninger J, Baumgartner ER. Partial 3-methylcrotonyl-CoA carboxylase deficiency in an infant with fatal outcome due to progressive respiratory failure. Eur J Pediatr 1998;157:225-229.

18. Darin N, Andersen O, Wiklund LM, Holmgren D, Holme E. 3-MethylcrotonylCoA carboxylase deficiency and severe multiple sclerosis. Pediatr Neurol 2007;36:132-134.

19. Dirik E, Yiş U, Paşaoğlu G, Chambaz C, Baumgartner MR. Recurrent attacks of status epilepticus as predominant symptom in 3-methylcrotonyl-CoA carboxylase deficiency. Brain Dev 2008;30:218-220.

20. Stucki M, Suormala T, Fowler B, Valle D, Baumgartner MR. Cryptic exon activation by disruption of exon splice enhancer: novel mechanism causing 3-methylcrotonyl-CoA carboxylase deficiency. J Biol Chem 2009;284:28953-28957.

21. Baumgartner MR. Molecular mechanism of dominant expression in 3-methylcrotonyl-CoA carboxylase deficiency. J Inherit Metab Dis 2005;28:301-309.

22. Baumgartner MR, Dantas MF, Suormala T, et al. Isolated 3-methylcrotonyl-CoA carboxylase deficiency: evidence for an allele-specific dominant negative effect and responsiveness to biotin therapy. Am J Hum Genet 2004;75:790-800.
23. Nyhan W, Barshop B, Al-Aqeel A. Atlas of Inherited Metabolic Diseases, 3rd edn. Hodder-Arnold: London, 2012.

24. Jacobsen BK, Eggen AE, Mathiesen EB, Wilsgaard T, Njølstad I. Cohort profile: the Tromso Study. Int J Epidemio/ 2012;41:961-967.

25. Tewhey R, Nakano M, Wang X, et al. Enrichment of sequencing targets from the human genome by solution hybridization. Genome Bio/ 2009;10:R116.

26. Li H, Durbin R. Fast and accurate long-read alignment with Burrows-Wheeler transform. Bioinformatics 2010;26:589-595.

27. DePristo MA, Banks E, Poplin R, et al. A framework for variation discovery and genotyping using next-generation DNA sequencing data. Nat Genet 2011;43:491-498.

28. Purcell S, Neale B, Todd-Brown K, et al. PLINK: a tool set for whole-genome association and population-based linkage analyses. Am J Hum Genet 2007;81:559-575.

29. Smith EN, Jepsen K, Arias AD, Shepard PJ, Chambers CD, Frazer KA. Genetic ancestry of participants in the National Children's Study. Genome Biol 2014;15:R22.

30. Venables W, Ripley B. Modern Applied Statistics with S, 4th edn. Springer: New York, 2010.

31. Cingolani $P$, Platts $A$, Wang le $L$, et al. A program for annotating and predicting the effects of single nucleotide polymorphisms, SnpEff: SNPs in the genome of Drosophila melanogaster strain w1118; iso-2; iso-3. Fly (Austin) 2012;6:80-92.

32. McLaren W, Pritchard B, Rios D, Chen Y, Flicek P, Cunningham F. Deriving the consequences of genomic variants with the Ensembl API and SNP Effect Predictor. Bioinformatics 2010;26:2069-2070.

33. Morscher RJ, Grünert SC, Bürer C, et al. A single mutation in MCCC1 or MCCC2 as a potential cause of positive screening for 3-methylcrotonyl-COA carboxylase deficiency. Mol Genet Metab 2012;105:602-606.

34. Pemberton TJ, Absher D, Feldman MW, Myers RM, Rosenberg NA, Li JZ. Genomic patterns of homozygosity in worldwide human populations. Am J Hum Genet 2012;91:275-292.

35. Broman KW, Weber JL. Long homozygous chromosomal segments in reference families from the Centre d'Etude du Polymorphisme Humain. Am J Hum Genet 1999;65:1493-1500.

36. Kirin M, McQuillan R, Franklin CS, Campbell H, McKeigue PM, Wilson JF. Genomic runs of homozygosity record population history and consanguinity. PLoS One 2010;5:e13996.

37. American College of Medical Genetics Newborn Screening Expert Group. Newborn screening: toward a uniform screening panel and system--executive summary. Pediatrics 2006;117(5 Pt 2):S296-\$307.

38. Wolfe LA, Finegold DN, Vockley J, et al. Potential misdiagnosis of 3-methylcrotonyl-coenzyme A carboxylase deficiency associated with absent or trace urinary 3-methylcrotonylglycine. Pediatrics 2007;120:e1335-e1340.

39. Lander ES, Botstein D. Homozygosity mapping: a way to map human recessive traits with the DNA of inbred children. Science 1987;236:1567-1570.

40. Sheffield VC, Carmi R, Kwitek-Black A, et al. Identification of a Bardet-Biedl syndrome locus on chromosome 3 and evaluation of an efficient approach to homozygosity mapping. Hum Mol Genet 1994;3:1331-1335.

(c) (i)

This work is licensed under a Creative Commons Attribution 3.0 Unported License. The images or other third party material in this article are included in the article's Creative Commons license, unless indicated otherwise in the credit line; if the material is not included under the Creative Commons license, users will need to obtain permission from the license holder to reproduce the material. To view a copy of this license, visit http:// creativecommons.org/licenses/by/3.0/ 